\title{
Layered polymeric optical systems using continuous coextrusion
}

Hyunmin Song, Kenneth Singer, Yeheng Wu, Juefei Zhou, Joe Lott, et al.

Hyunmin Song, Kenneth Singer, Yeheng Wu, Juefei Zhou, Joe Lott, James Andrews, Anne Hiltner, Eric Baer, Christoph Weder, Robert Bunch, Richard Lepkowicz, Guy Beadie, "Layered polymeric optical systems using continuous coextrusion," Proc. SPIE 7467, Nanophotonics and Macrophotonics for Space Environments III, 74670A (9 September 2009); doi: 10.1117/12.830035

SPIE Event: SPIE Optical Engineering + Applications, 2009, San Diego, California, United States 


\title{
Layered Polymeric Optical Systems Using Continuous Coextrusion
}

\author{
Hyunmin Song ${ }^{\mathrm{a}}$, Kenneth Singer*b, Yeheng $\mathrm{Wu}^{\mathrm{b}}$, Juefei Zhou ${ }^{\mathrm{b}}$, Joe Lott ${ }^{\mathrm{a}}$, James Andrews ${ }^{\mathrm{c}}$, Anne \\ Hiltner ${ }^{\mathrm{a}}$, Eric Baer ${ }^{\mathrm{a}}$, Christoph Weder ${ }^{\mathrm{a}, \mathrm{e}}$, Robert Bunch $^{\mathrm{d}}$, Richard Lepkowicz ${ }^{\mathrm{d}}$, Guy Beadie ${ }^{\mathrm{f}}$ \\ ${ }^{\mathrm{a}}$ Dept. of Macromolecular Science and Engineering, Case Western Reserve University, 2100 \\ Adelbert Rd, Cleveland, OH, USA 44106 \\ ${ }^{b}$ Dept. of Physics, Case Western Reserve University, 13900 Euclid Ave, Cleveland, OH USA 44106 \\ ${ }^{c}$ Dept. of Physics and Astronomy, Youngstown State University, Youngstown, OH 44555 \\ ${ }^{\mathrm{d}}$ Dept. of Physics, Rose-Hulman University, Terre Haute, IN 47803 \\ 'Adolphe Merkle Institute and Fribourg Center for Materials, University of Fribourg, 1700 Fribourg, \\ Switzerland \\ fOptical Sciences Division, Naval Research Lab, Washington, DC 20375
}

\begin{abstract}
Polymers are receiving considerable attention as components in novel optical systems because of the tailored functionality, ease of manufacturing, and relatively low cost. The processing of layered polymeric systems by coextrusion is a method to produce films comprising hundreds to thousands of alternating layers in a single, one-step roll-to-roll process. Several layered polymer optical systems have been fabricated by coextrusion, including gradient refractive index lenses, tunable refractive index elastomers, photonic crystals, and mechanically tunable photonic crystals. Layered polymeric optical systems made by coextrusion can also incorporate active components such as photoreactive additives for multilayered patterning and laser dyes for all-polymer laser systems. Coextrusion is a process which allows for the flexible design of polymeric optical systems using layers with thickness spanning the nanoscale to the microscale.
\end{abstract}

Keywords: Coextrusion, polymer photonic crystal, refractive index of multilayered films, polymer dye laser

\section{INTRODUCTION}

Polymers are rapidly emerging as the next generation materials for novel optical systems because of the ability to tailor their properties, low cost, and ease of processing[1]. Layered polymer systems doped with active media such as dyes or nanoparticles have become integral tools in manipulating and controlling light for data storage[2], sensors and sensory devices[3], lenses[4,5], optical switches and limiters[6], efficient light emitters[7], and waveguides[8,9]. Melt processing by coextrusion allows for quick roll-to-roll manufacturing of high volumes of polymer film producing cost-competitive, layered optical systems.

Coextrusion, a solvent-free method, employs layer multipliers that assemble hundreds to thousands of alternating layers of two or more dissimilar polymers in a continuous process[10]. The thickness of the layers can span several microns to tens of nanometers and the volume composition of each component can be controlled using melt pumps and die design. The polymer melt viscosity is an important parameter to obtain uniform layers and stable melt flow. Processing two or more polymers with the same melt viscosity at a given temperature is pivotal for the successful fabrication of multilayered films. The multilayer coextrusion process itself has been introduced by the Dow Chemical Company 40 years ago[11] and has been greatly refined since[4,7,10,12]. It is commercially used, for example, by $3 \mathrm{M}$ to produce reflective films[13].

Discussed here are several layered optical systems that were recently developed and rely on multilayer co-extrusion, including gradient refractive index (GRIN) optics, functional one-dimensional photonic crystals, all-polymer lasers, and photopatternable films. Material selection and processing allows precise control over refractive index allowing the fabrication of refractive index patterns, including gradients and superlattices. The periodic dielectric nature leads to interference effects characteristic of Bragg gratings and photonic crystals. Doping with active species allows for

Nanophotonics and Macrophotonics for Space Environments III, edited by Edward W. Taylor, David A. Cardimona, Proc. of SPIE Vol. 7467, 74670A · ( 2009 SPIE · CCC code: 0277-786X/09/\$18 · doi: 10.1117/12.830035 
optoelectronic functionality. All of these characteristics suggest that multilayer polymer films can make important contributions to future photonic devices.

\section{INDEX OF REFRACTION AGILE MATERIALS THROUGH MULTIPLAYERING}

\subsection{Gradient Refractive Index Optics}

Incorporating a gradient refractive index (GRIN) in a lens allows for an extra degree of freedom (much like an aspheric surface) in optical design[14], and has important implications in both imaging and non-imaging systems. In imaging applications the GRIN is used to correct for aberrations, thus achieving the same or better imaging quality as homogeneous optical systems with fewer elements. In non-imaging systems the GRIN is used to redistribute the rays in a nonlinear fashion in order to achieve the desired output. Examples of both an imaging and non-imaging system designed with spherical GRIN lenses will be presented below, but first the fabrication method of polymer spherical GRIN lenses will be discussed.

Inspired by nature, a new class of polymer optics with a spherical GRIN profile that mimics structures found in biological systems has been developed using continuous coextrusion[4,5]. The building block for the spherical GRIN lens is multilayered polymers that have a controllable index of refraction and can be easily shaped and molded together. For the lenses discussed in this article the specific multilayered film has an overall thickness of 50 microns and consists of 4096 layers of alternating poly(styrene-co-acrylonitrile) with $17 \mathrm{wt} \%$ acrylonitrile (SAN17) and polymethlymethacrylate (PMMA). The individual layer thickness of the overall film is on the order of $10 \mathrm{~nm}$, thus resulting in an effective medium[15] meaning that light travels through the multilayered structure as if the system was one material, and consequently, a single refractive index. PMMA has an index of refraction of 1.49 while SAN17 has an index of refraction of 1.57 (both at a wavelength of $633 \mathrm{~nm}$ ), which gives a dynamic range of index of refraction of 0.08 . The refractive index of the multilayered film can be shifted towards the high index polymer or the low index polymer depending on the relative thickness of the PMMA layer to the SAN17 layer. Individual films were fabricated in steps of index of refraction of 0.002 resulting in 51 unique films that can be used in the fabrication process of spherical GRIN lenses.

A spherical GRIN lens was designed to minimize on-axis aberrations in an $F / 2.25$ plano-convex singlet for imaging applications. The lens was optimized in the lens design package Zemax ${ }^{\circledR}$, and a $4^{\text {th }}$ order polynomial was used for the GRIN profile. The optimized GRIN profile goes linearly from 1.53 at the curved surface to 1.57 at the plano-surface. To fabricate the lens a stack with the desired GRIN profile was assembled from the multilayer films. This stack was then fused into one sheet under heat and pressure near the glass transition temperature of the polymers. This stack is then molded to a spherical shape, and the top surface is polished flat. The process described is outlined in Figure 1.

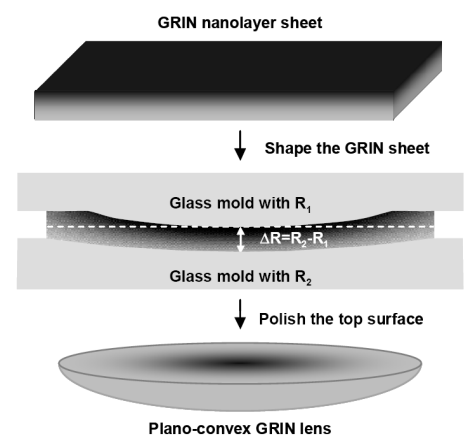

Figure 1. Fabrication of plano-convex GRIN lenses from nanolayered GRIN sheets.

The imaging properties of the spherical GRIN lens are quantified by measuring its modulation transfer function (MTF). The MTF was measured using an incoherent edge resolution method[16] as well as a coherent point spread method. The experimental procedure is discussed in more detail in[5], but the primary results are shown in Figure 2a. It is clear from these results that the spherical GRIN lens is superior to the homogeneous glass lens under both tests. Neither the GRIN nor homogeneous lens is corrected for multiple wavelengths so the imaging with the single 
wavelength laser source is slightly better for both lenses. The difference in imaging performance is apparent when looking at a magnified Air Force resolution chart as shown in Figure $2 b$.

(a)

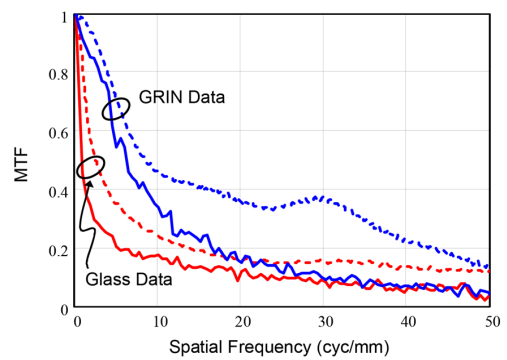

(b)

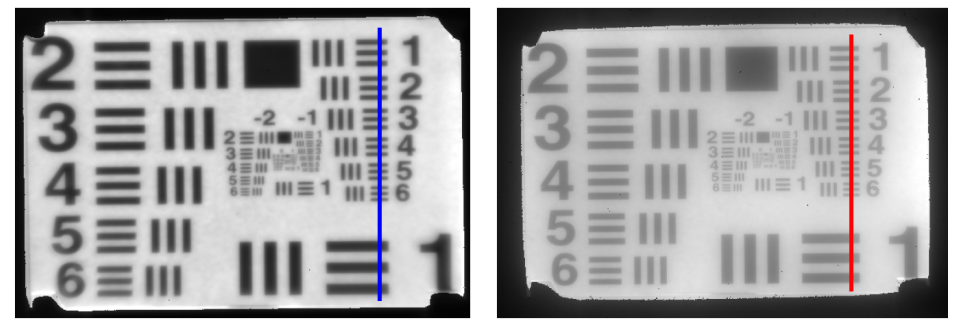

Figure 2. Characterization of GRIN lenses (a) Modulation transfer function (MTF) of a GRIN lens (blue) compared to a commercial glass lens (red). The solid lines were extracted from an edge analysis of an image chart (indicated by the red and blue line in the Air Force resolution chart) and the dashed lines were extracted from a focused HeNe laser beam. The CCD had a pixel pitch of $(8.53 \times 9.85)$ microns. The units of the horizontal axis correspond to the spatial frequency of the camera. (b) Air Force resolution chart seen through a GRIN lens (left) and image is an Air Force resolution chart seen through a commercial glass lens (right).

Spherical GRIN lenses with the GRIN contours decoupled from the surface have been investigated for the non-imaging application of laser beam shaping[17]. We examined two element beam shapers, which take an input Gaussian laser beam and transforms it into a uniform irradiance flat top. Beam shaping systems are traditionally designed using aspheric lenses[18], which makes this a good test for spherical GRIN optical elements. The designed system features high transmission efficiency, with nearly $100 \%$ of the energy transferred to the output beam and a variance of less than $3 \%$ in uniformity from the center to the edge of the beam, which is shown in Figure 3. Additionally, the optical path remains constant from the center of the beam to the edge of the so it remains collimated.

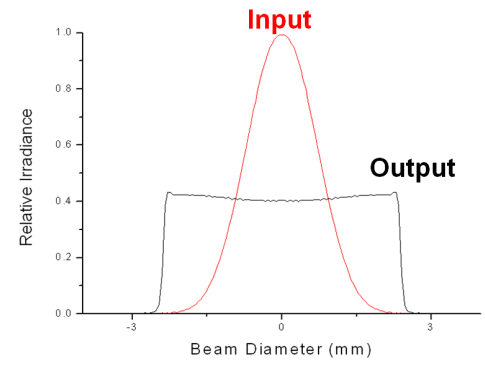

Figure 3. Profile of Gaussian input and flat top output.

(a)

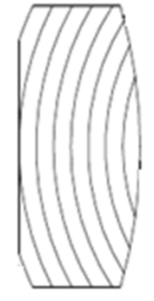

(b)

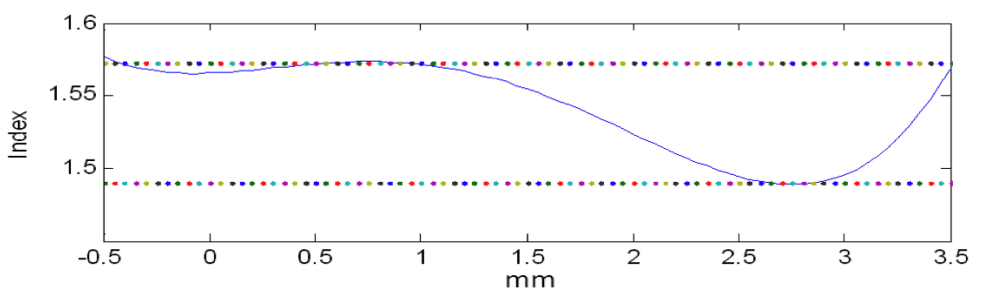

Figure 4. (a) Decoupled spherical GRIN lens for beam shaping applications. (b) On axis index of refraction profile of first element of beam shaper. 
One feature of spherical GRIN lenses as applied to beam shapers is of particular importance. In layered GRIN lens the largest ray deviation will occur when the gradient terminates at a surface. Orienting the curvature of the gradient in the opposite direction of the curvature of the lens surface allows the system to deviate rays at a maximum while spreading the beam. A visual representation of a decoupled GRIN lens is shown in Figure 4a. This novel GRIN structure can be fabricated through the co-extrusion process mentioned previously, and a combination of polishing and diamond turning. The on-axis GRIN profile for the first element is shown in Figure $4 \mathrm{~b}$, and is seen to be highly complex.

In conclusion, we have described two successful implementations of a spherical GRIN polymer lens for optical applications. For the imaging application we successfully reduced to practice a spherical GRIN lens operating at F/2.25 that had superior performance to that of homogeneous lens. We have also designed a laser beam shaping system that is compact has energy transfer and uniformity comparable to that of asphere based systems.

\section{LAYERED POLYMERIC PHOTONIC CRYSTALS}

A photonic crystal is a material with a periodic variation in the refractive index over a length scale of the quarter wavelength of light. This variation produces an optical bandgap, which prohibits a range of frequencies from propagating through the material. The magnitude of the reflectivity and the spectral width of the bandgap depend on the index contrast between the layers, layer thickness homogeneity, and number of layers. . For a planar layered material consisting of alternating layers of two polymers in an A/B fashion the center wavelength $\left(\lambda_{c}\right)$ of the stop band can be defined as:

$$
\lambda_{\mathrm{c}}=2\left(\mathrm{n}_{1} \mathrm{~d}_{1}+\mathrm{n}_{2} \mathrm{~d}_{2}\right) ; \quad \Delta \lambda=4 \lambda_{\mathrm{c}} \Delta \mathrm{n} / \pi\left(\mathrm{n}_{1}+\mathrm{n}_{2}\right)
$$

where $\lambda_{\mathrm{c}}$ is the wavelength of the first order reflection of the electromagnetic wave, $\mathrm{n}_{1} \mathrm{~d}_{1}$ the optical thickness material A (optical thickness is defined as the product of the refractive index, $n$, and the physical layer thickness, $d$ ) and $n_{2} d_{2}$ the optical thickness of material B. The spectral width of the reflection band, $\Delta \lambda$, is related to the difference in refractive index, $\Delta \mathrm{n}$, between the two polymers. Photonic crystals can be used as light filters, waveguides, and reflectors. Melt processable photonic crystals were first realized by Alfrey, Schrenk and Gurnee[11]. In this section, the effects of defects in layered photonic crystals, angular effects in 1-D photonic crystals, and the effect of $\Delta \mathrm{n}$ on the reflective properties of layered photonic crystals, and layered elastomeric photonic crystals are discussed. In the later sections, photonic crystals serve as the basis for active optical layered systems, such as information storage and lasers and lightemitting sources.

\subsection{Layered Glassy PS and PMMA Photonic Crystals $(\Delta n=0.090)$}

Although convenient and cost-effective, coextrusion by layer multiplication is not a perfect process, and as a result, layered photonic crystals produced by this method show considerable layer thickness variation, some leading to socalled defect states in the reflection band[19]. We have studied how this layer variation affects the reflective properties of photonic crystals in 128 layers of PS and PMMA. These materials were chosen for their high $\Delta \mathrm{n}$, high transparency, and low interfacial thickness during melt processing[20].

Figure 5a depicts a cross-section of a 128 layered PS/PMMA photonic crystal film imaged by AFM. The average layer thickness was measured to be $86 \mathrm{~nm}$ with a standard deviation of $24 \%$. The histogram in Figure $5 \mathrm{~b}$ shows the layer thickness distribution in these layered photonic crystals. The thickness distribution is not Gaussian which suggests a systematic error in layer thickness rather than randomly distributed errors. The majority of the layers lie between 80 to $90 \mathrm{~nm}$. The reflectivity of the multilayered film is measured indirectly using transmission. From Figure 5c, the transmission of PS/PMMA photonic crystal film is about 5\% between 538 and $562 \mathrm{~nm}$ (half peak band width of $25 \mathrm{~nm}$ ), or about $95 \%$ reflection of green light. However, the model spectrum obtained from transfer matrix calculations[21] predicts $100 \%$ reflection. Moreover, the model spectrum does not show the numerous side bands observed in the PS/PMMA multilayered films. For further insight, the transfer matrix model was modified with the actual thickness distribution of the film measured from an AFM cross-section image, which was compared to the transmission spectrum of the PS/PMMA multilayered film shown in Figure 5d. This model better describes the data. .

The appearance of side bands in multilayered PS/PMMA photonic crystals arises from the distributions in layer thickness complicating the interference between forward and backward moving waves. Although most of the reflectivity occurs between 538 and $562 \mathrm{~nm}$, the disorder-induced side bands in the high energy region, between 400 and $530 \mathrm{~nm}$ 
arise from the small thickness regions of the layer thickness distribution. This effect can be partly explained by the coextrusion process, where layers that are close to the extruder walls exhibit a different melt flow and shear rate than layers near the center of the multipliers, and thus become thinner than predicted. Interested readers can understand the details of flow instabilities in coextrusion from work done by Schrenk and co-workers[22], Han and Shetty[23], and Macosko and co-workers[24].
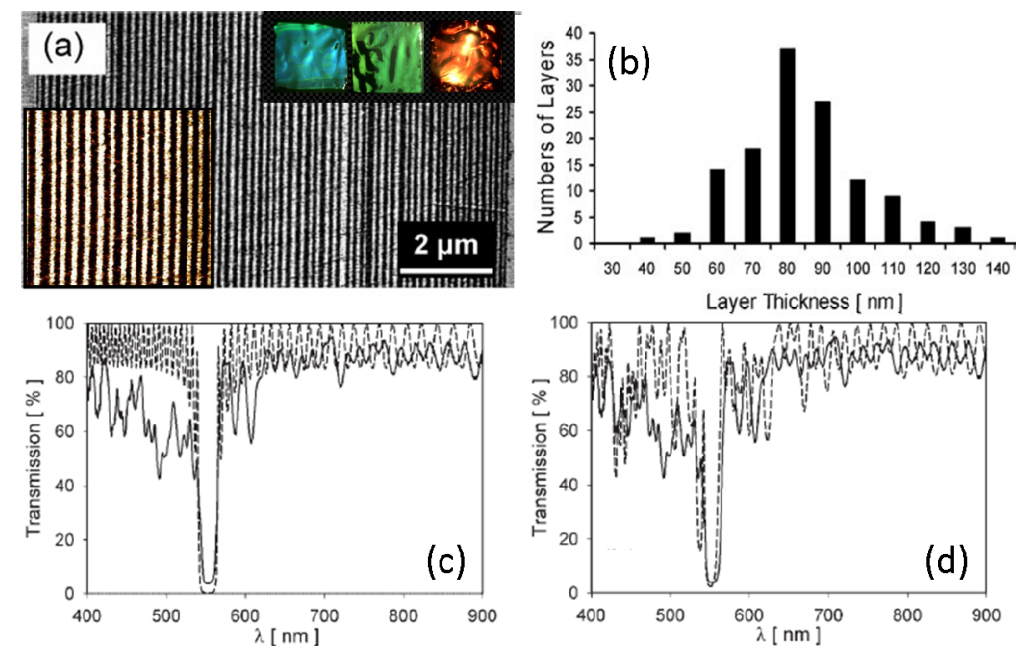

Figure 5. Characterization of 128 layered PS/PMMA photonic films. (a) Cross-section of a 128 layered PS/PMMA photonic film using AFM. The bright layers are PMMA and the dark layers are PS. The lower left inset shows the layers at a higher magnification with each layer approximately $86 \mathrm{~nm}$. The upper right inset shows the reflective properties of photonic crystals. (b) Histogram detailing the layer thickness distribution of PS/PMMA photonic films measured by AFM. (c) Transmission spectrum of 128 layered PS/PMMA photonic film (solid line) compared to a model spectrum (dashed line) using a transfer matrix calculation with $0 \%$ std. dev. (d) Transmission spectrum of 128 layered PS/PMMA photonic film (soild line) compared to a model spectrum which has incorporated a standard deviation of $24 \%$ in each layer thickness.

\subsection{Effect of $\Delta \mathrm{n}$ on the Reflective Properties of Multilayered Films}

By altering the polymers used in the multilayer process it is possible to perform a systematic study of the optical properties of the photonic crystal as a function of $\Delta \mathrm{n}$. The layered system described in Section 3.1 is fabricated from PS/PMMA multilayers having a $\Delta \mathrm{n}=0.090$. However, by employing polystyrene $(\mathrm{n}=1.585)$ and a poly(vinylidiene fluoride-co-hexafluoropropylene) having $15 \mathrm{wt} \%$ hexafluoropropylene (PVDF-HFP15) melt blended with PMMA $(80 / 20 \mathrm{wt} \%$ PVDF-HFP15/PMMA, $\mathrm{n}=1.421)$, the $\Delta \mathrm{n}$ can be increased to 0.164. PVDF and PMMA are miscible polymers and the inclusion of HFP in PVDF greatly decreases its crystallinity[25,26]. Only $20 \mathrm{wt} \%$ PMMA blended into PVDF-HFP20 is required to obtain an amorphous, transparent blend having a refractive index of 1.421 .
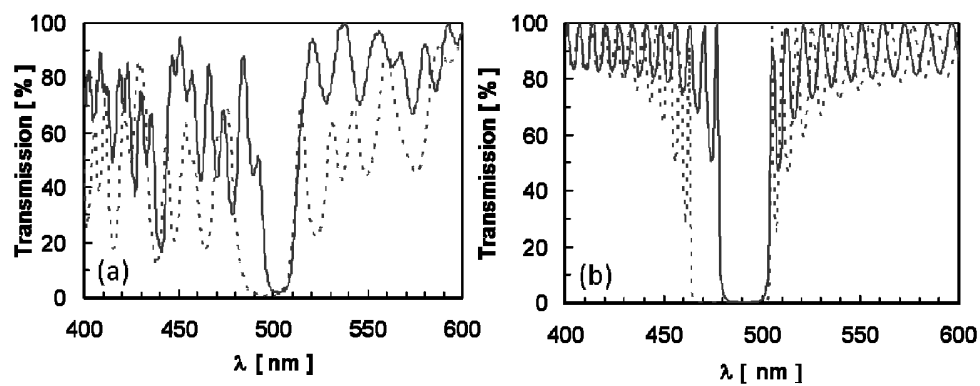

Figure 6. Effect of $\Delta \mathrm{n}$ on photonic crystal properties of multilayered films. (a) 128 layer PS/PMMA system (solid line) compared against a 128 layer PS/(PVDF-HFP/PMMA) system (dashed line). (b) Modeling of a system having 128 layers and a $\Delta \mathrm{n}$ of 0.090 (solid line) and a $\Delta \mathrm{n}$ of 0.164 (dashed line). 
The $\Delta \mathrm{n}$ between the two layered polymers has a large effect on its photonic crystal properties. Figure 6a depicts a 128 layered system using PS/PMMA having a $\Delta \mathrm{n}$ of 0.090 (solid line) compared to a 128 layered system using PS against an $80 / 20 \mathrm{wt} \%$ PVDF-HFP15/PMMA blend having a $\Delta \mathrm{n}$ of 0.164 (dashed line). The system having a $\Delta \mathrm{n}$ of 0.164 has a bandwidth of $33 \mathrm{~nm}$ for the main reflection peak. However, the system having a $\Delta \mathrm{n}$ of 0.090 has a bandwidth of only 18 $\mathrm{nm}$. This agrees with the theoretical prediction from Equation (1). Furthermore, the system having high $\Delta \mathrm{n}(0.164)$ also shows increased reflection from the side bands. By increasing the $\Delta \mathrm{n}$ of the layered photonic crystals, not only does the bandwidth widen, but also the reflections are more intense. The effect of $\Delta \mathrm{n}$ has also been modeled. Figure $6 \mathrm{~b}$ shows modeling of a 128 layered system having a $\Delta \mathrm{n}$ of 0.090 (solid line) and a $\Delta \mathrm{n}$ of 0.164 (dashed line). The modeling predicts a $43 \mathrm{~nm}$ bandwidth for the $\Delta \mathrm{n}=0.164$ system and a bandwidth of $25 \mathrm{~nm}$ for the $\Delta \mathrm{n}=0.090$ system, both of which are much wider than the experimental observations. This can be attributed to the layer thickness distributions which are inherent to the coextruded systems.

\subsection{Soft, Tunable Photonic Crystals using PU/PEBAX Elastomers}

Using a pair of elastomeric polymers, the layer thickness can be tuned by the strain, thereby changing the position of the reflection band[13]. The possibility of fabricating tunable photonic crystals was explored using thermoplastic polyurethane (TPU, $\mathrm{n}=1.55$ ) layered against Pebax (poly(amide-b-ether), $\mathrm{n}=1.48$ ). The soft tunable system has a $\Delta \mathrm{n}$ of 0.07 .
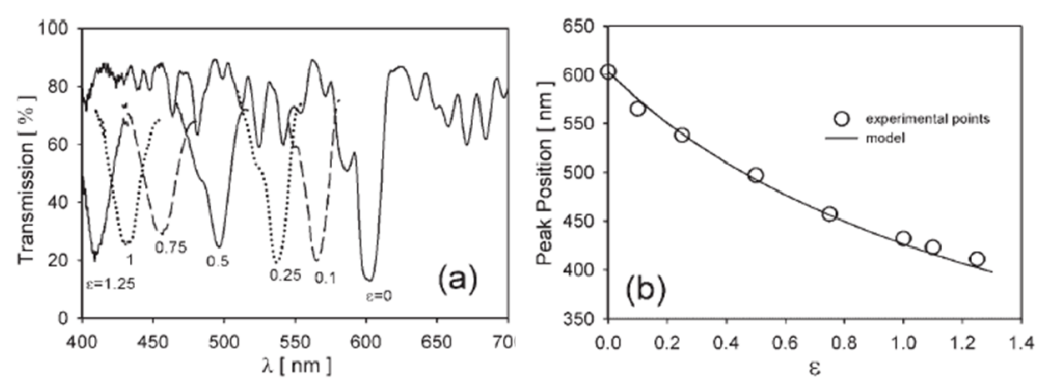

Figure 7. Effect of strain, $\varepsilon$, on the photonic properties of multilayered films. (a) Transmission spectra 128 layers of $\mathrm{PU} /$ Pebax with strain ranging from 0 to $125 \%$. (b) Peak reflection position obtained from experimental results (open circles) compared to a calculated peak reflection position (solid line) using Equation 2.

The transmission spectra of a 128 layered PU/Pebax film with strains $(\varepsilon)$ from 0 to $125 \%$ were collected and shown in Figure 7a. The reflection band shifts from $600 \mathrm{~nm}(\varepsilon=0)$ to $415 \mathrm{~nm}(\varepsilon=125 \%)$, spanning nearly the entire visible spectrum. The peak reflection position can be described as:

$$
\lambda=\frac{\lambda_{0}}{\sqrt{1+\varepsilon}}
$$

Where $\lambda$ is the peak reflection position, $\lambda_{\mathrm{o}}$ is the reflection position at $\varepsilon=0$, and $\varepsilon$ is the strain. This equation assumes that the volume is conserved and the refractive index change of the elastomer layer does not contribute to wavelength shifting during stretching. In Figure 7b, the peak reflection position is plotted against the strain $\varepsilon$ and compared against the calculated peak reflection position using Equation 2. There is excellent agreement between the experimental results and the model predictions. Elastomeric photonic crystals may be useful for producing sensitive strain sensors or tunable reflectors for various optical systems.

\section{ALL-POLYMER LASERS}

\subsection{Distributed Bragg Reflector (DBR) Lasers}

Polymers containing active laser dyes are gaining considerable attention because of their broad tailorability of properties and their ease of processing vis-à-vis inorganic semiconductor materials. To build an all-polymer laser, reflective resonators were fabricated using 128 layers of PS/PMMA photonic crystal films[7,12]. The gain layer was a polymer doped laser dye and was sandwiched between two polymer resonators. Such a lasing structure is known as a surfaceemitting distributed Bragg reflector laser (DBR) as seen in Figure 8a. Using the DBR architecture, all-polymer lasers 
have been successfully demonstrated using three laser dyes, rhodamine 6G (R6G), pyyromethene 580 (P580), and 1,4bis-( $\alpha$-cyano-4-methoxystyryl)-2,5-dimethoxy-benzene (C1RG). Both R6G and Pyrromethene 580 are commercially available, however, $\mathrm{C} 1 \mathrm{RG}$ was synthesized[27]. Figures $8 \mathrm{~b}$ and $8 \mathrm{c}$ depict laser action in DBR lasers with the gain media as C1RG and R6G blended into PMMA. In both cases, a strong lasing mode is observed. The DBR lasers are optically pumped using a nanosecond Nd:YAG laser. Details of the optical properties of the DBR lasers are seen in Figures 8d and 8e. The multilayered reflectors are designed such that the absorption of the gain media is outside of the main reflection band and the peak fluorescence of the gain media lies at the center of the reflection band. The optical pump is now transparent through the DBR structure and can efficiently excite the gain medium.
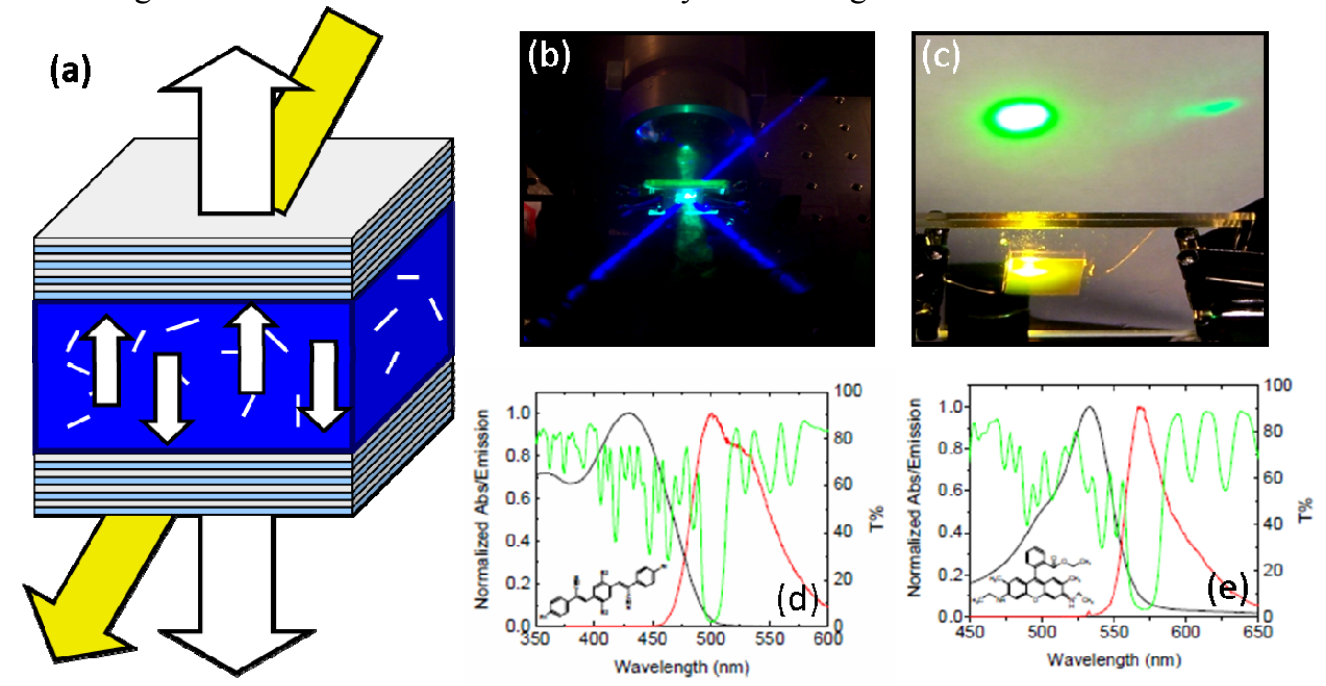

Figure 8. DBR polymer laser. (a) Structure of DBR laser and mechanism of laser action. The small white arrows depict fluorescence resonating in the structure. The large white arrows indicate laser action. The large yellow arrow shows oblique angle optical pumping of the DBR laser using a Nd:YAG source. (b) Picture of laser action of C1RG DBR laser. (c) Picture of laser action of R6G DBR laser. (d) Laser design criteria for C1RG showing absorption of the dye (black line), fluorescence of the dye (red line) and the transmission of the photonic reflectors (green line) (e) Laser design criteria for R6G showing absorption of the dye (black line), fluorescence of the dye (red line) and the transmission of the photonic reflectors (green line).

films. Emission occurs when gain exceeds losses within the cavity reflection band. Typical emission spectra are shown in Figure 9. The thickness of the dielectric layer determines the spacing between lasing modes as:

$$
\Delta \lambda=\lambda^{2} /\left(2 \mathrm{n}_{\mathrm{eff}} \mathrm{L}_{\mathrm{eff}}\right)
$$

where $\Delta \lambda$ is the mode spacing, $\lambda$ is the wavelength at the center of the reflection band, $\mathrm{n}_{\mathrm{eff}}$ is the effective refractive index of the dielectric layer, and $\mathrm{L}_{\text {eff }}$ is the effective cavity length. It is found that this effective length includes

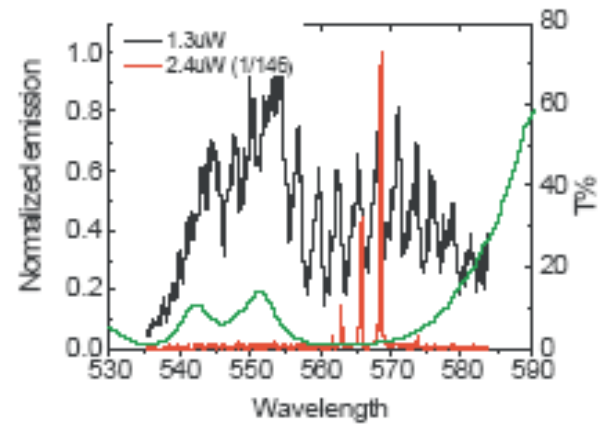

Figure 9. Lasing modes in a R6G DBR laser. The black line shows fluorescence below threshold and the red line shows laser action above threshold. The green line shows the reflection band of the multilayered structure. 
contributions from the mode penetration into the DBR reflectors, and that the variation in layer thickness increases the penetration into the multilayer films[28]. Figure 9 shows the lasing spectrum of a R6G DBR laser. There are 3 laser modes whose mode spacing can be calculated from Equation 3. The threshold was measured to be $90 \mu \mathrm{J} / \mathrm{cm}^{2}$.

The performance of the DBR laser depends on the polymer host matrix as well as the laser dye. Table 1 shows a summary of the DBR lasers fabricated and their performance[29]. A 50/50 PVDF-HFP/PMMA blend showed the best results of laser performance, obtaining 50\% optical efficiency and a low threshold of $35 \mu \mathrm{J} / \mathrm{cm}^{2}$ compared to the PMMA host polymer.

Table 1: Summary of DBR Performance Results

\begin{tabular}{l|cccccc}
\hline $\begin{array}{c}\text { Host } \\
\text { Polymer }\end{array}$ & $\begin{array}{c}\text { Gain } \\
\text { Medium } \\
\text { Thickness } \\
(\boldsymbol{\mu \mathbf { m } )})\end{array}$ & Laser Dye & $\begin{array}{c}\text { Molar Density } \\
(\mathbf{M})\end{array}$ & $\begin{array}{c}\text { Pump } \\
(\mathbf{n m})\end{array}$ & $\begin{array}{c}\text { Slope } \\
\text { Efficiency } \\
(\mathbf{\%})\end{array}$ & $\begin{array}{c}\text { Threshold } \\
\text { Fluence } \\
\left(\boldsymbol{\mu} \mathbf{J} / \mathbf{c m}^{2}\right)\end{array}$ \\
\hline $50 / 50$ & 53 & Pyrromethene 580 & $1.9 \times 10^{-2}$ & 520 & 50 & 35 \\
PVDF/PMMA & 135 & C1-RG & $1.6 \times 10^{-3}$ & 430 & 14 & 435 \\
PMMA & 40 & R6G & $5.4 \times 10^{-3}$ & 532 & 19 & 90 \\
PMMA & \multicolumn{7}{c}{}
\end{tabular}

\subsection{Distributed Feedback (DFB) Lasers}

Distributed feedback (DFB) lasers are processed directly from coextrusion to produce lasers on a large-scale continuous process $[29,30]$. The mechanism and structure for DFB lasing differs from DBR lasing. First, the dye is doped directly into one of the polymer layers during the extrusion process, rather than using a thick dielectric layer in a sandwich structure. Secondly, the DFB structure comprises both the gain and feedback media. A detailed theory of DFB lasing in photonic crystals can be found in reference [31]. The structure of the DFB laser can be seen in Figure 10a. The structure comprises 128 layers of poly(styrene-co-acrylonitrile) containing $25 \mathrm{wt} \%$ acrylonitrile (SAN25, $\mathrm{n}=1.56$ ) doped with about $1 \mathrm{wt} \%$ R6G laser dye and fluoroelastomer THV $(\mathrm{n}=1.37)$, which is a terpolymer consisting of tetrafluoroethylene (TFE), hexafluoropropylene (HFP), and tetrafluoroethylene (TFE). The total film thickness is $12 \mu \mathrm{m}$. In a perfect feedback structure, emission occurs at the band edge, where the photon density of states is a maximum and the group velocity is a minimum.

(a)
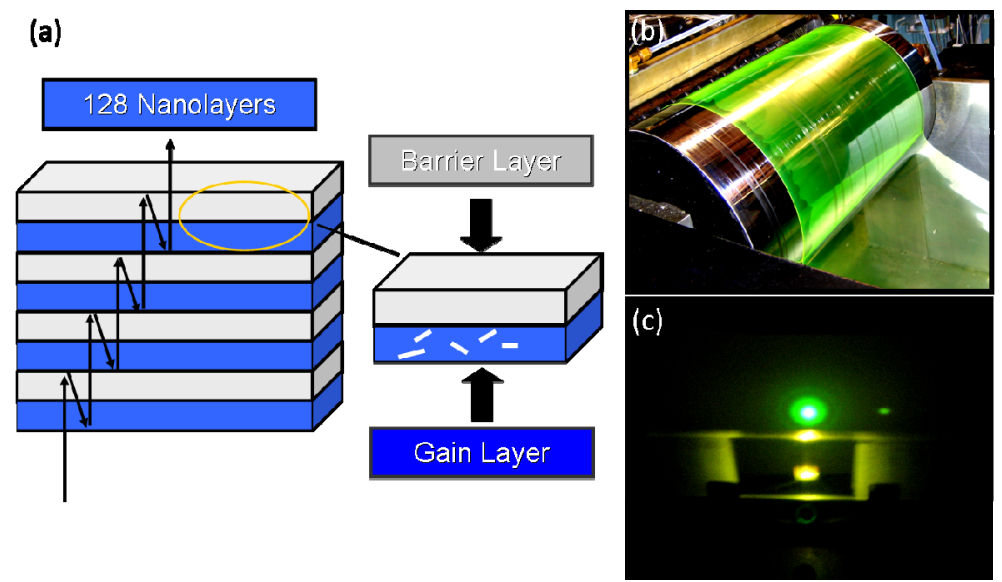

Figure 10. Concept of the DFB laser. (a) Light passing through a DFB structure. The light resonates in the multilayer structure. (b) Roll-to-roll processing of DFB laser. (c) DFB laser action showing a strong laser mode (large spot) and the pumping mode (small spot).

The advantage of DFB lasing is the possibility of producing laser film on a roll-to-roll process. Figure 10b shows a picture of a DFB laser doped with C1RG being collected on the chilled take-up roll. The films require no post-treatment. 
Laser film can be taken directly from the extrusion process to be further characterized. Figure 10c shows laser action of a DFB laser doped with R6G dye showing a strong laser mode.
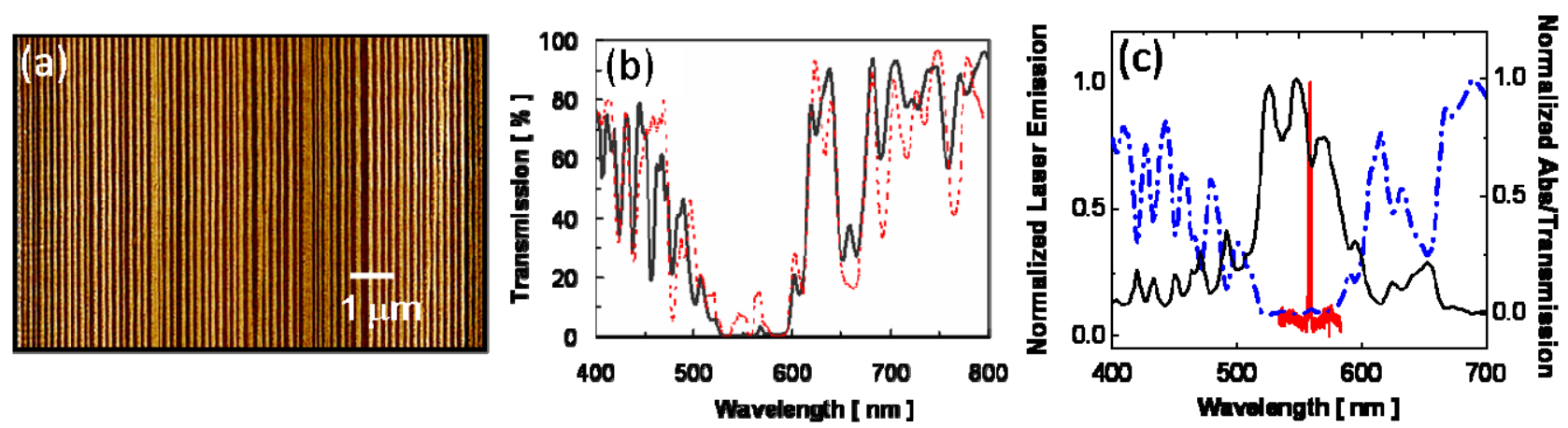

Figure 11. Characterization of DFB lasers. (a) AFM cross-section of a 128 layer SAN25/THV DFB laser. The layers were measured to be approximately $95 \pm 25 \mathrm{~nm}$. The bright layers are THV and the dark layers are SAN25. (b) Using AFM thickness distributions, the transmission spectrum is reconstructed via modeling (dashed red line) and compared to the experimental results (solid line). (c) DFB laser action (redline) shown with respect to the transmission spectrum (dashed blue line) and the transmission spectrum shown in absorbance units (solid black line).

To further study how the layer structure influences the lasing properties, the layer thickness distribution of 128 layer SAN25/THV DFB laser film was obtained from an AFM cross-section image (Figure 11a). The layers were measured to be $95 \pm 25 \mathrm{~nm}$. The transmission spectrum using transfer matrix modeling was compared to the experimental transmission spectrum. Figure $11 \mathrm{~b}$ shows the layered structure influencing the transmission spectrum of the film by contributing numerous defect-induced side bands. The defects also extend inside the main reflection band. Figure 11c shows the transmission spectrum (blue line) compared to the same transmission spectrum in logarithmic absorbance units (solid black line) showing the details of the reflection band, The spectra in $11 \mathrm{c}$ are all normalized. Lasing occurs at a defect state within the reflection band (red line) rather than at the band edge. The lasers investigated displayed a $2.6 \%$ optical efficiency and a threshold of $238 \mu \mathrm{J} / \mathrm{cm}^{2}$.

Fabrication of DFB lasers also requires the need for a barrier layer, which prevents the laser dye from diffusing throughout the system reducing the effective absorption of the SAN25 doped dye gain layer by dilution. In Figure 12a-b, THV is shown to be an excellent barrier to dye molecules, under melt conditions. The optical micrographs clearly show the distinction between the fluorescing SAN25+dye layers and the transparent THV layers.
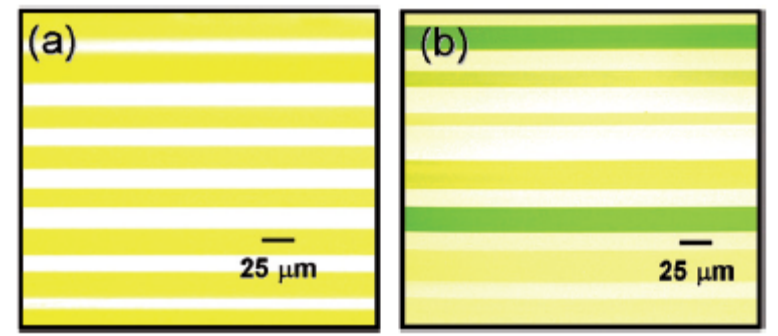

Figure 12. The barrier properties of coextruded films containing SAN25+dye and THV. (a) OM micrograph of a 64 layered SAN25 doped with $1 \mathrm{wt} \%$ R6G against THV. The yellow layers show the fluorescence of R6G in SAN25. (b) OM micrograph of a 64 layered SAN25 doped with $1 \mathrm{wt} \% \mathrm{C} 1 \mathrm{RG}$ against THV. The colored layers show the fluorescence of C1RG.

\section{PHOTOPATTERNABLE FILMS}

Multilayered optical interference is directly governed by the refractive index of the materials employed. This refractive index can be tuned by incorporating photoreactive additives, which upon sufficient excitation, undergo a chemical 
reaction capable of changing the refractive index of the material[32]. The materials chosen to produce coextruded photopatternable films are PMMA $(\mathrm{n}=1.490)$ and a photoreactive additive trans-cinnamic acid $(\mathrm{CA}, \mathrm{n}=1.555)$. The multilayered structure consists of 1024 layers. CA readily undergoes $2+2$ cycloaddition, where species of CA dimerize when photoactivated (Figure 13a). CA shows 3 different crystal forms, $\alpha, \beta$, and $\gamma$. Dimerization of the $\alpha$ - and $\beta$ - form lead to $\alpha$-truxinic acid (2) and $\beta$-truxillic acid (3). A concentration of $12 \mathrm{wt} \%$ CA was melt blended into PMMA prior to extrusion. Although higher concentrations can be used to obtain larger refractive index changes, CA was found to plasticize PMMA and $12 \mathrm{wt} \%$ was the highest concentration that can be used for coextrusion conditions. Blending CA into PMMA also increased the refractive index. Blends of $12 \mathrm{wt} \%$ CA into PMMA have a refractive index of 1.502.
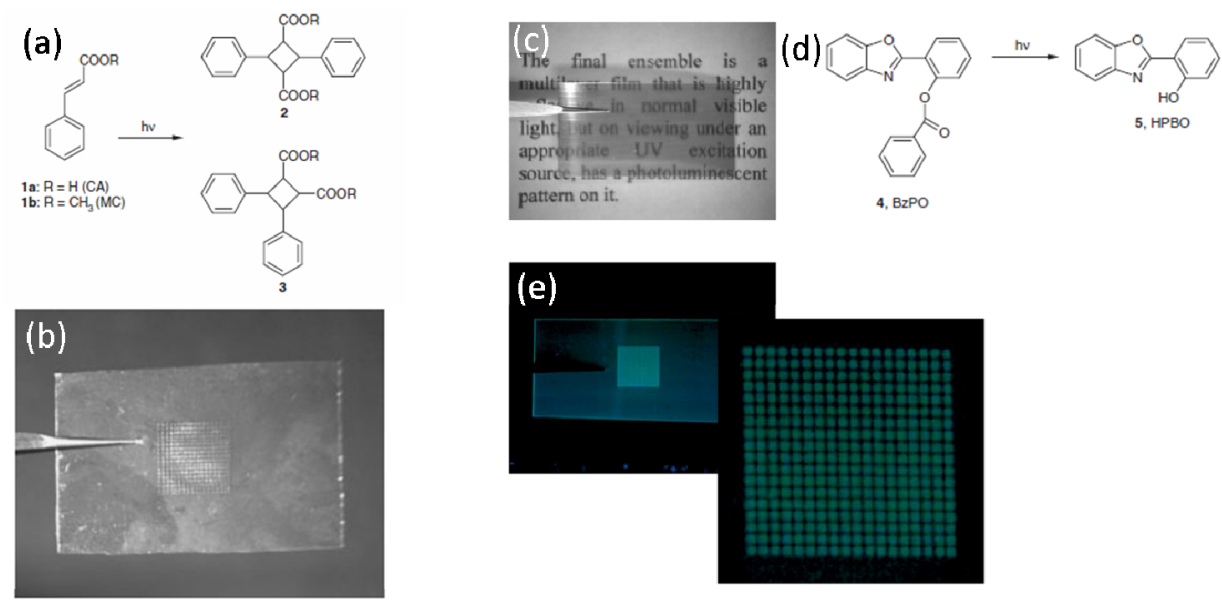

Figure 13. Depiction of photopatterned films using a refractive index changing additive and a fluorescent dye. (a) Photodimerization of CA into (2) truxinic acid and (3) truxillic acid. (b) Picture of a photopatterned coextruded film containing PMMA layered against PMMA with $12 \mathrm{wt} \% \mathrm{CA}$. (c) Photograph of a 1024 layered PMMA/BzPO-PC film containing $0.5 \mathrm{wt} \%$ BzPO exposed to $254 \mathrm{~nm}$ UV light through a checkerboard mask for $15 \mathrm{~min}$. (d) Photochemically induced cleavage of 2-(2'-benzoylphenyl)benxoaxazole (BzPO) under formation of 2-(2'-hydroxyphenyl)benzoxazole (HPBO). (e) 1024 layered PMMA/BzPO-PC film shown under $365 \mathrm{~nm}$ UV light. The inset shows higher magnification.

After extrusion, the multilayered film was exposed to $254 \mathrm{~nm}$ UV radiation and was masked with a checkerboard pattern. The areas exposed to the UV light changed refractive index of the PMMA+CA layers as a result of photodimerization from $\mathrm{n}=1.511$ to $\mathrm{n}=1.502$ and the refractive index of the unexposed areas remained the same $(\mathrm{n}=1.511)$. A $\Delta \mathrm{n}$ of 0.009 was enough to see a reflective checkerboard pattern on the film as seen in Figure 13b. The change in refractive index of the CA+PMMA layers can be described using the Lorentz-Lorenz equation[33]:

$$
R=(M / \rho)\left(n^{2}-1\right)\left(n^{2}+2\right)
$$

where $\mathrm{R}$ is the molar refraction, $\mathrm{M}$ is the molecular weight, $\rho$ is the density, and $\mathrm{n}$ is the refractive index. With the values of $\rho$ and $\mathrm{n}$ for CA $\left(1.2475 \mathrm{~g} \mathrm{~cm}^{-1}, 1.555\right)$ and PMMA $\left(1.19 \mathrm{~g} \mathrm{~cm}^{-1}, 1.490\right)$, it becomes clear that the substantial increase in refractive index when CA is blended into PMMA is not only related to the high polarizability of the CA additive, but also that the irradiation of UV light reduced the refractive index of the CA+PMMA layers. This is consistent with the consumption of CA to produce dimers of lower molar refraction.

Another method to producing photopatterns in multilayered films is to use a fluorescence mechanism, rather than a change in refractive index. To produce fluorescent patterns, a multilayered film of polycarbonate $(\mathrm{PC}, \mathrm{n}=1.591)$ doped with $0.5 \mathrm{wt} \%$ 2-(2'-benzoylphenyl)benzoaxazole (BzPO) was layered against PMMA. Figure 13c shows a picture of a 1024 layered PC-BzPO with $0.5 \mathrm{wt} \% \mathrm{BzPO}$ and PMMA multilayered film. The film is reflective, however no pattern is visibly seen. BzPO is not a fluorescently active species. After exposure to UV light, however, the ester bond is cleaved, and $\mathrm{BzPO}$ transforms into 2-(2'-hydroxyphenyl)benzoxazole (HPBO) radical, which converts into HPBO by hydrogen abduction from the environment and is an active fluorescent species (Figure 13d). After exposure to $254 \mathrm{~nm}$ UV light with a checkerboard mask, the film is brilliantly fluorescent using $354 \mathrm{~nm}$ excitation (Figure 13e). The areas exposed to 
the $254 \mathrm{~nm}$ UV radiation fluoresce, and the areas unexposed remain transparent. These photoactivated multilayered films can be used for applications involving security features and identification marks.

\section{CONCLUDING REMARKS}

Mutlilayered coextrusion is a useful process to produce polymeric materials with interesting optical properties from a continuous melt process. Multilayered films can have modulated refractive indices for adaptive optics, beam steering and switching. The refractive index can also be controlled using stacks of multilayered films to generate a gradient refractive index profile, which is useful for producing compact, lightweight lenses. Photonic crystals and tunable photonic crystals having strong reflection bands can be fabricated. These photonic crystals can have increased functionality by doping the layers with various additives such as photoreactive molecules and fluorescently active laser dyes. Some interesting future prospects include fabricating tunable laser systems, changing the optical pump from a high power Nd:YAG source to a low power laser diode, and producing film capable of writing in 3-dimensions for high volume storage. Coextrusion promises a flexible, low-cost manufacturing process for a new generation of photonic devices.

\section{ACKNOWLEDGEMENTS}

The authors are grateful to the National Science Foundation for financial support under the Science and Technology Center for Layered Polymer Systems (CLiPS) under grant number 0423914.

\section{REFERENCES}

[1] Samuel, I. D. W. and Turnbull, G. A. "Organic semiconductor lasers." Chem. Rev. 107, 1272-1295 (2007).

[2] Li, L., Ling, Q. D., Lim, S. L., Tan, Y. P., Zhu, C., Chan, D. S. H., Kang, E. T. and Neoh, K. G. "A flexible polymer memory device." Organic Electronics. 8, 401-406, (2007).

[3] Kunzelman, J., Crenshaw, B.R., and Weder, C.; Self-assembly of chromatic dyes: A new mechanism for humidity senors. J. Mater. Chem. 17, 2989-2991, (2007).

[4] Jin, Y., Tai, H., Hiltner, A., Baer, E., Shirk, J. S. "New class of bioinspired lenses with a gradient refractive index." J. of Appl. Poly. Sci. 103, 1834-1841, (2007).

[5] Beadie, G., Shirk, J. S., Rosenberg, A., Lane, P. A., Fleet, E., Kamdar, A. R., Jin, Y., Ponting, M., Kazmierczak, T., Yang, Y., Hiltner, A., and Baer, E. "Optical properties of a bio-inspired gradient refractive index polymer lens." Optics Express. 16(15), (2008).

[6] Kang, J. W., Kim, E., and Kim, J. J. "All-optical switch and modulator using photochromic dye doped polymer waveguides." Optical Materials. 21, 543-548, (2002).

[7] Singer, K.D., Kazmierczak, T., Lott, J., Song, H., Wu, Y., Andrews, J., Baer, E., Hiltner, A., and Weder, C. "Toward Roll-to-Roll Production of Polymer Microresonator Lasers." Opt. and Photonics News. 19, 27-28 (2008).

[8] Ma, H., Jen, A. K. Y., and Dalton, L. R. "Polymer-based optical waveguides: Materials, processing, and devices." Adv. Mater. 14(19), (2002).

[9] Tien, P. K., and Ulrich R. "Theory of Prism-Film Coupler and Thin-Film Light Guides." J. of the Opt. Soc. of Am. 60(10), (1970).

[10] Mueller, C. D., Nazarenko, S., Ebeling, T., Schuman, T. L., Hiltner, A., and Baer, E. "Novel structures by microlayer coextrusion." Polymer Engineering and Science. 37, 355-362 (1997).

[11] Alfrey, T. Jr., Gurnee, E. F., and Schrenk, W. J. "Physical optics of iridescent multilayered plastic films." Poly. Eng. and Sci. 9(6), (1969).

[12] Singer, K. D., Kazmierczak, T., Lott, J., Song, H., Wu, Y., Andrews, J., Baer, E., Hiltner, A., and Weder, C. "Meltprocessed all-polymer distributed Bragg reflector laser." Optics Express. 16(14), (2008).

[13] Weber, M. F., Stover, C. A., Gilbert, L. R., Nevitt, T. J., and Ouderkirk, A. J. "Giant birefrigent optics in multilayered polymer mirrors." Science. 287, (2000).

[14] Moore, D. "Gradient-index optics: a review," Appl. Optics 19, 1035-1038 (1980). 
[15] Reynolds, J.A. Hough, J.M. "Formulae for dielectric constant of mixtures," Proc. Phys. Soc. London B 70, 769-775 (1957).

[16] J.W. Goodman, Introduction to Fourier Optics (McGraw-Hill, San Francisco, CA, 1968).

[17] R. Zahreddine, R. M. Bunch, R.S. Lepkowicz, E. Baer, A. Hiltner, "Beam shaping with spherical GRIN lenses," Proc. SPIE 7062, 706214 (2008).

[18] J. A. Hoffnagle, C. M. Jefferson, "Design and performance of a refractive optically system that converts a Gaussian to a flattop beam," Appl. Opt. 39(20), 5488-5499 (2000).

[19] Kazmierczak, T., Song, H., Hiltner, A., and Baer, E. "Polymeric one-dimensional photonic crystals by continuous coextrusion." Macromol. Rapid Commun. 28, 2210-2216, (2007).

[20] Higashida, N., Kressler, J., Yukioka, S., Inoue, T. "Ellipsometric measurements of positive c parameters between dissimilar polymers and their temperature dependence." Macromolecules. 25, 5259-5262, (1992).

[21] Vasicek, A. "Optics of thin films." 1st ed, North-Holland Publishing Co, Netherlands, Amsterdam, (1960).

[22] Schrenk, W. J., Bradley, N. L., Alfrey, T. Jr., Maack, H. "Interfacial flow instability in multilayered coextrusion."

Poly. Eng. and Sci. 18(8), (1978).

[23] Han, C. D., and Shetty, R. "Interfacial instability in flat film coextrusion." Poly. Eng. and Sci. 18(3), (1978).

[24] Zhang, J., Lodge, T. P., and Macosko, C. W. "Interfacial slip reduces polymer-polymer adhesion during coextrusion." J. Rheol. 50(1), 41-57, (2006).

[25] Nishi, T., and Wang, T. T. "Melting point depression and kinetic effects of cooling on crystallization in polyvinylidene fluoride-polymethacrylate mixtures." Macromolecules. 8(6), (1975).

[26] Pimbert, S., Avignon-Poquillon, L., Levesque, G. Calorimetric study of fluorinated methacrylic and vinyl polymer blends." Polymer. 43, 3295-3302, (2002).

[27] Löwe, C. and Weder, C. "Synthesis and properties of photoluminescent 1,4-bis-(a-cyano-4methoxystyryl)benzenes." Synthesis. 9, 1185-1190 (2002).

[28] Wu, Y., Singer, K. D., Petschek, R. G., Song, H., Baer, E., and Hiltner, A. "Mode delocalization in 1D photonic crystal lasers." Submitted for publication.

[29] Zhou, J., Singer, K. D, Lott, J., Song, H., Wu, Y., Andrews, J., Baer, E., Hiltner, A, and Weder, C. "All-polymer distributed feedback and distributed Bragg reflector lasers produced by roll-to-roll layer multiplying coextrusion."

Submitted for publication.

[30] Song, H., Singer, K. D., Lott, J., Wu, Y., Zhou, J., Andrews, J., Baer, E., Hiltner, A., and Weder, C. "Continuous melt processing of all-polymer distributed feedback lasers." J. Mater. Chem. DOI:10.1039/b909348f (2009).

[31] Dowling, J. P., Scalora, M., Bloemer, M. J., Bowden, C. M. "The photonic band edge laser: A new approach to gain enhancement." J. Appl. Phys. 75(4), (1994).

[32] Tangirala, R., Baer, E., Hiltner, A., and Weder, C. "Photopatternable reflective films produced by nanolayer extrusion." Adv. Funct. Mater. 14(6), (2004).

[33] Meeten, G. H. "Optical properties of polymers." Elsevier Applied Science, London and New York, (1989). 